UN IVERSITY OF COPENHAGEN

\title{
Gait as evidence
}

Lynnerup, Niels; Larsen, Peter Kastmand

Published in:

IET Biometrics

DOI:

10.1049/iet-bmt.2013.0090

Publication date:

2014

Document version

Publisher's PDF, also known as Version of record

Citation for published version (APA)

Lynnerup, N., \& Larsen, P. K. (2014). Gait as evidence. IET Biometrics, 3(2), 47-54. https://doi.org/10.1049/ietbmt.2013.0090 


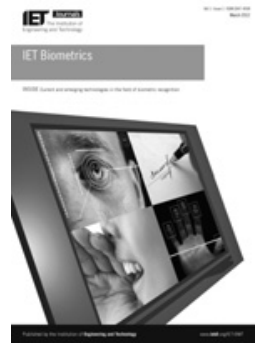

ISSN 2047-4938

\title{
Gait as evidence
}

\author{
Niels Lynnerup, Peter Kastmand Larsen \\ Unit of Forensic Anthropology, Department of Forensic Medicine, University of Copenhagen, Frederik d. V's Vej 11, \\ DK-2100 Copenhagen, Denmark \\ E-mail: nly@sund.ku.dk
}

\begin{abstract}
This study examines what in Denmark may constitute evidence based on forensic anthropological gait analyses, in the sense of pointing to a match (or not) between a perpetrator and a suspect, based on video and photographic imagery. Gait and anthropometric measures can be used when direct facial comparison is not possible because of perpetrators masking their faces. The nature of judicial and natural scientific forms of evidence is discussed, and rulings dealing with the admissibility of video footage and forensic evidence in general are given. Technical issues of video materials are discussed, and the study also discusses how such evidence may be presented, both in written statements and in court.
\end{abstract}

\section{Introduction}

The subject of study in a forensic anthropological analysis is the whole body. It may constitute any bodily features which are visible or may be deduced from imagery techniques. Primarily, such features comprise facial features and general bodily features of height and body build, but may also include features such as tattoos, wrinkles on the dorsal aspect of the fingers, venous markings on arms and pathological features such as acne or wounds and lesions (e.g. [1]).

The body in motion, specifically gait, is also a very visible feature, which may also be captured on video [2]. The perpetrators are often masked in robberies so as to preclude the use of facial features or other of the above-mentioned features, but as the perpetrators necessarily have to move around and enter and exit a bank, their gait, posture and stance may be captured on video. Furthermore, even though masked, height as well as other bodily measures (e.g. height to eyes, shoulder height and stride length) may be derived photogrammetrically from the video images (e.g. [3]).

At our department, we have specifically focused on gait analyses and bodily measures ascertained by photogrammetry as bodily features to be included when matching perpetrators to suspects.

\section{Nature of the evidence}

The use of the above-mentioned features has to be underpinned by proper scientific research. Scientific evidence may be viewed as the result of scientific studies, which can be placed a hierarchy, denoting the strength of the evidence [4]. Thus, a case report is generally viewed as the entry level step, followed by randomised, blind studies, and finally, as the highest step, review studies based on a multitude of blind trials [4]. In a gait analyses setting, this could thus equal first case stories noting how a specific gait, or several gait features, could be used for finding a match (e.g. [5]), followed by laboratory studies where gait recognition is tested on a larger population [6], and finally, when several such tests have been carried out, summarised in critical review studies. It is of course implicit for all these steps that they comprise peer-reviewed case stories and studies, accessible for scientific peers.

The (natural) scientific meaning of evidence, or proof of a method, is not the same as the judicial meaning. Even novel methods are not necessarily contested in court. Rather, it has often been the case that a judge may allow scientific evidence, simply because the person presenting it is a scientist. While this is so in Denmark, other countries have, following more recent court rulings, begun to specifically define what may constitute scientific evidence, or expert evidence. In the UK, in a ruling in 2011 (R. v. Smith) the following was stated: (i) it is for a judge to decide who is a competent expert in a particular field; (ii) it is essential for the proper administration of justice that there are independent persons expert (in fingerprint examination); (iii) a competent scientist must keep detailed notes of his examination and the reasons for his conclusions; and (iv) modern methods of presentation of expert evidence should be used to make evidence accessible to jury and save court time [7]. It is perhaps the last two clauses which are of special relevance in the kind of analyses discussed here. Gait evidence often relies on the study of live motion picture capture for gait traits to be evaluated and presented as indicative of a match or not. Secondly, one may note how there is a certain 'rapprochement' to the scientific notion of evidence in stating that notes of examination must be kept: in other words, it is not enough for a scientific expert to state his or her opinion; the opinion must be backed by notes, presumably (although not directly stated) so as to allow another expert to arrive at the same conclusions (or at least infer how the first expert arrived at his or her opinions). 


\section{www.ietdl.org}

Probably, the most well-known ruling about expert scientific evidence is the US ruling in the case Daubert v. Merrell Dow Pharmaceuticals [8]. Often simply called the Daubert rules (or the Daubert trilogy, following two further US Supreme Court rulings), these constitute a non-exclusive four part test for the admissibility of scientific evidence. For a given scientific method to be used in court it is important to consider: (i) whether the expert's methodology can or has been tested; (ii) whether it has been subject to peer review and publication; (iii) its known or potential error rate and the existence and maintenance of standards controlling its operation; and (iv) whether it has attracted widespread acceptance within the relevant scientific community [8].

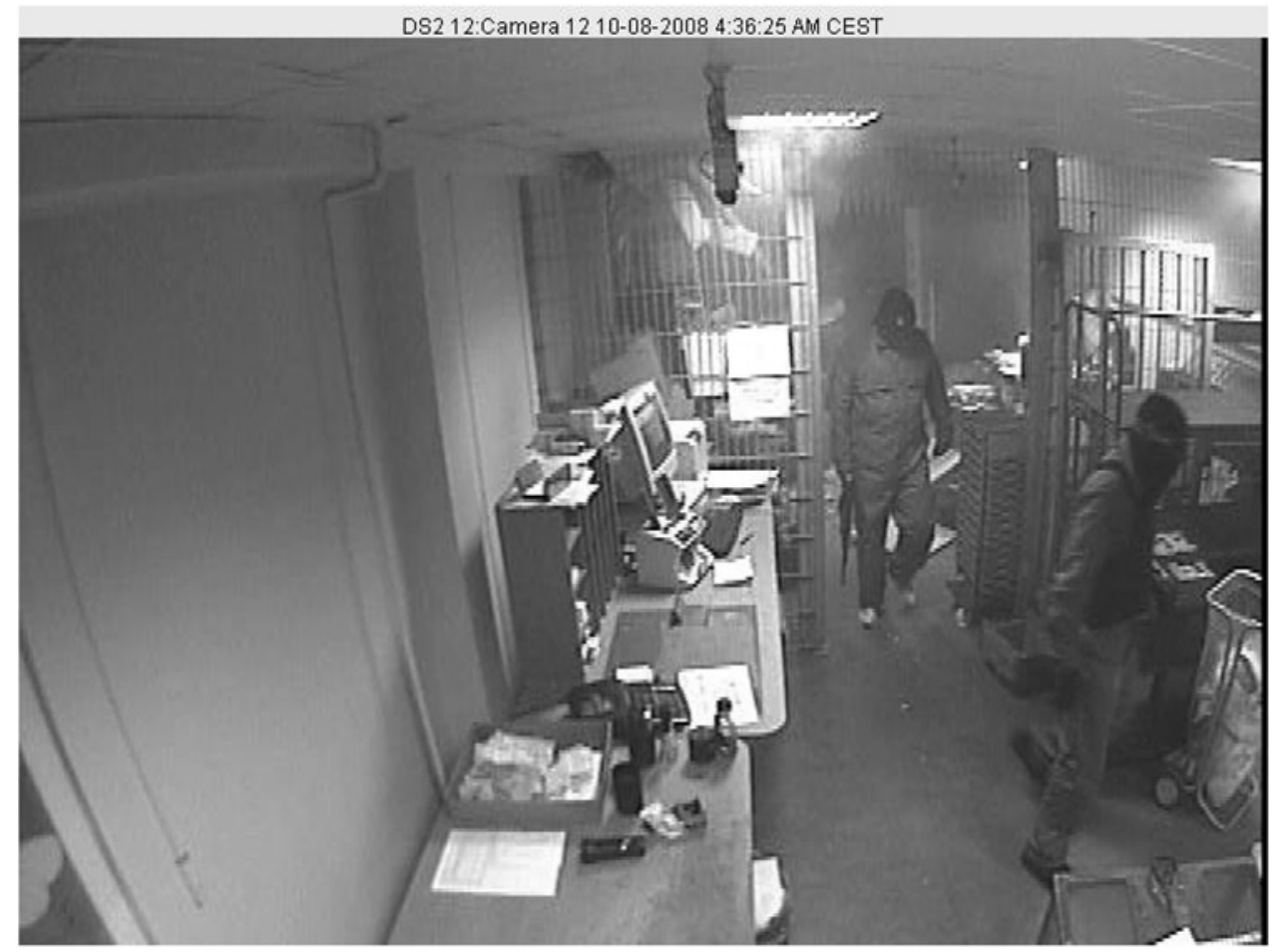

a

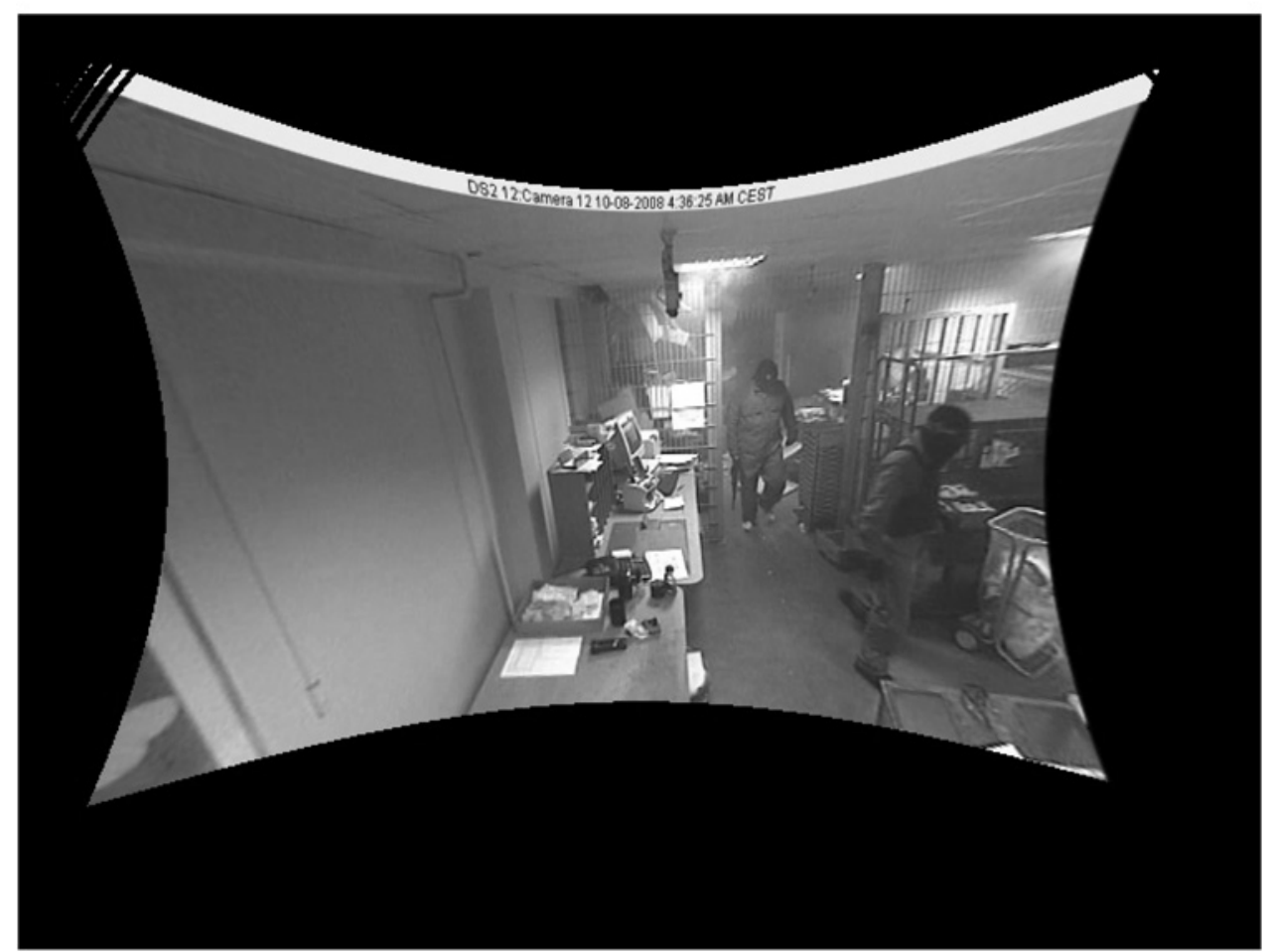

$b$

Fig. 1 Technical issues involved in using CCTV or video footage

$a$ and $b$ Image from a CCTV camera before and after application of correction of lens distortion

It will be seen that if an uncorrected image is used, the lens distortion may result in errors in photogrammetry or assessment of joint angles (and thus gait) 
Obviously, the last clause may be difficult if the scientific method is rather novel, or may be difficult to replicate in other laboratories, perhaps because of high costs or very specialised equipment. Still, the Daubert rules in our opinion serve as an excellent guide for what one should aim at when employing gait and photogrammetry as evidence.

Another UK ruling (in 2012) may also be of some import for gait and photogrammetric evidence. In R. v. Barnes reverse projection evidence was critically assessed, and the judge found the evidence admissible, as reverse projection was 'Not new science, but employed photographic techniques', and that 'Because the expert was applying techniques with which he was familiar, all that was required was the production of film which could provide a fair and accurate comparison with the crime scene recording' [9]. Thus, some aspects of photogrammetry may be viewed not as novel scientific hypotheses, but rather as tested techniques.

Photogrammetric and gait analyses are thus in our opinion admissible as evidence. However, it is important to heed the above guidelines in making sure that technical issues and error ranges are addressed properly and presented so that the statements derived from these analyses, may be deemed valid by the court for a given case.

\section{Technical issues}

It is important to be aware of the technical issues involved in using CCTV or video footage. It is most often digital, which usually means of rather high technical quality (although there may be proprietary compression formats which may mean that the captured frame rate may be low).

One can differentiate between intrinsic and extrinsic factors which have a bearing of the technical quality:

Extrinsic factors:

- How the camera is mounted, view angle, lighting etc.

- Obstructions in the camera line-of-sight.

\section{Intrinsic factors:}

- Camera specifications (lens, focusing, pixels, frame rate).

- Image storage (compression, proprietary file formats).

- Player specifications, including compression formats (pixels, frame rate).

We have found that much attention must be paid to the cameras and the image player systems. It is not trivial how the lens of a camera may distort the image, and how different file compression formats work, and indeed how the player displays the files (e.g. the player may automatically adjust to a given screen dimension, which may again distort the images). One way to ascertain lens distortion is to use software which allows corrections (see Fig. 1). As for the other intrinsic errors it is important to always include the measuring of some fixed length or height in the field of view, as a simple test as to whether measures inferred from the footage actually match static, defined measures in the actual setting (Fig. 2).

After having considered there factors, the variability of ascertaining measuring points in order to estimate stature or other lengths, should also be addressed (e.g. [10]). A recent experimental example is determining the errors associated

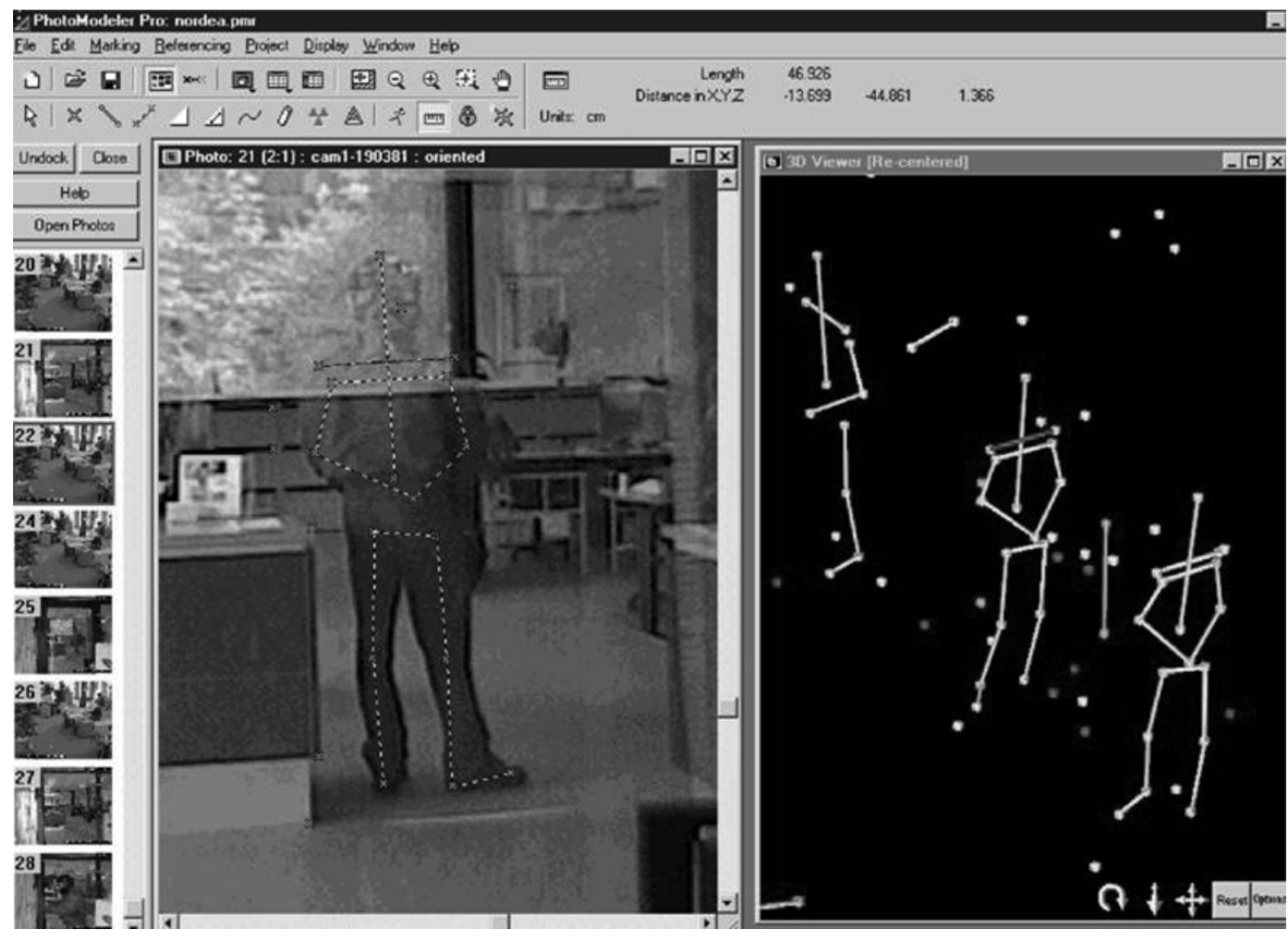

Fig. 2 Measurement control

Aside measuring several bodily features (seen as dotted lines on the perpetrator in the CCTV image and yellow lines in the 3D visualisation), the corner height of a counter is also measured, and compared with the physical measurement in situ (the counter is just to the left of the perpetrator, and is seen as the green line in the $3 \mathrm{D}$ visualisation) 
with determining eye height [11]. We performed some test trials on this measure, as eye height might be an obtainable measure in cases where a perpetrator is masked. The masking, for example, a balaclava or motorcycle helmet, will add to the total height (cephalic vertex), thus making it more difficult to directly compare with a maskless suspect. It has been our experience that the eyes are not so often masked, and thus might be an excellent 'marker'. However, in order to use this measure, we not only determined how eye height was related to total height and throughout a gait cycle, but also how the position of the head might impact on the eye height. Based on our results for 16 test subjects, we concluded that eye height varied by $\pm 4.3 \mathrm{~cm}$, regardless of total height, throughout the gait cycle, and that eye height varies between -13.6 and $+6.9 \mathrm{~cm}$, depending on whether the head is flexed fully forward or backward, respectively [11]. These measures can then be included in reports, where we measure the eye height, thus providing the error range of

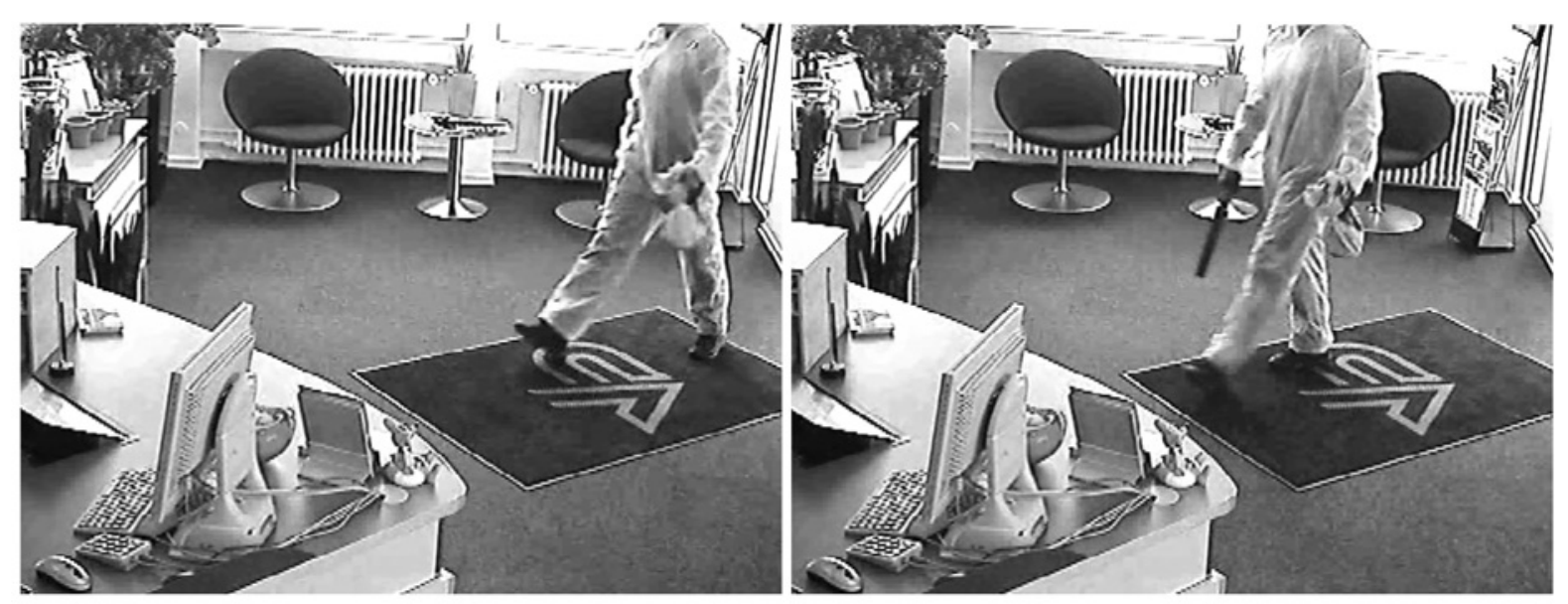

a

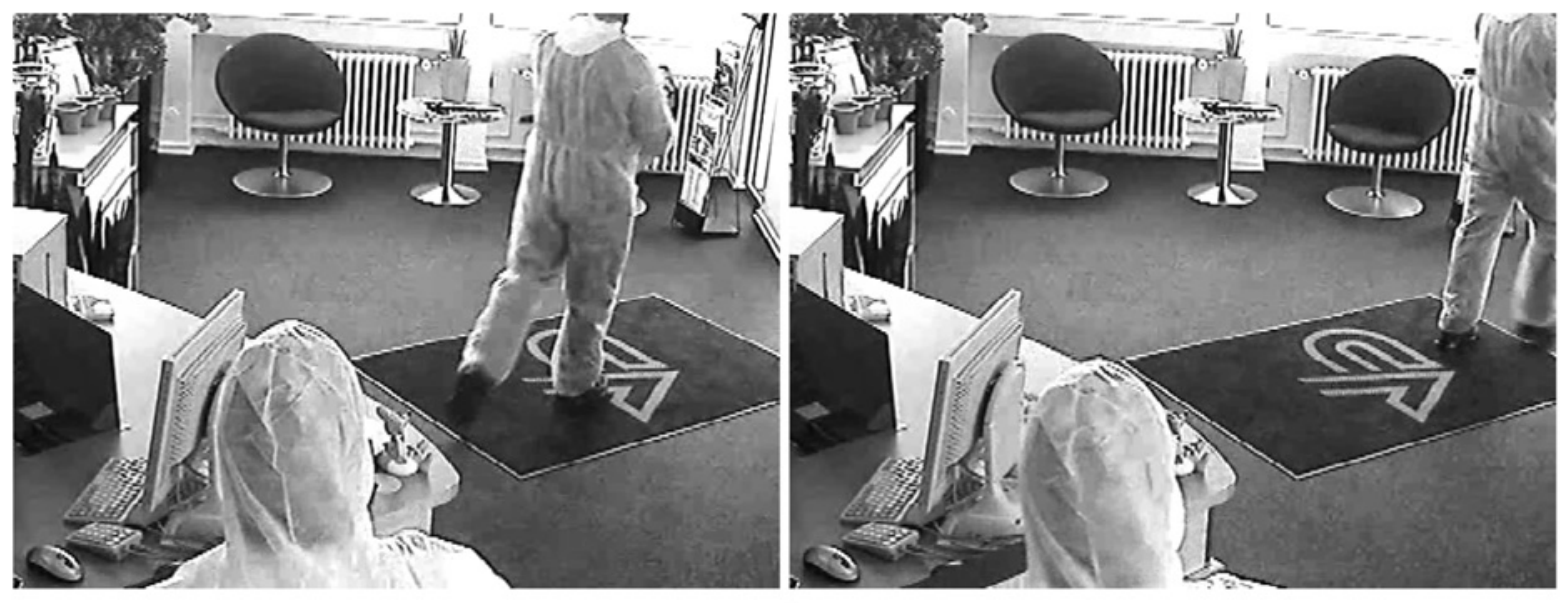

$b$

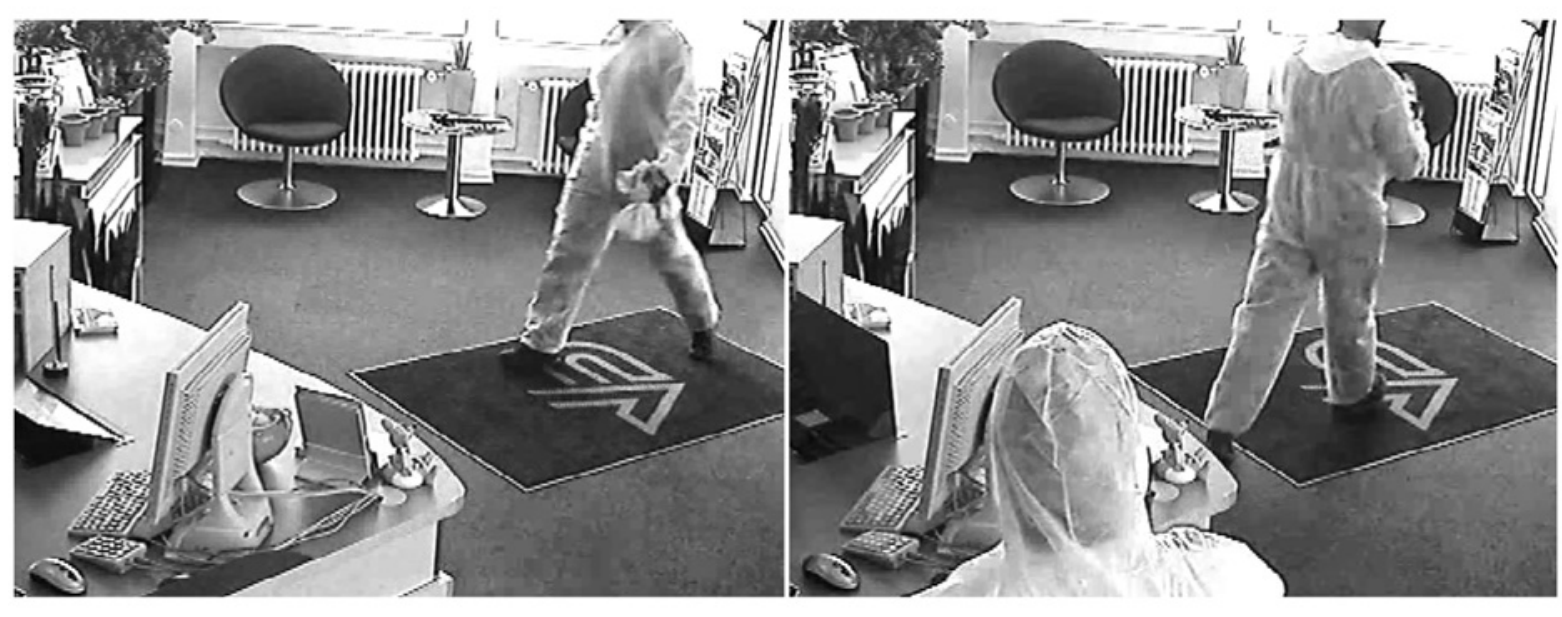

$c$

Fig. 3 Two images from video footage from a bank CCTV

$a$ Footage was used to state that the degree and form of the lower leg forward extension before heel-strike, was congruent to that of the suspect (not shown) $b$ Footage was used to state that the degree of outward rotation of the foot in the stance phase, as seen from behind, was congruent to that of the suspect $c$ Footage was used to state that a relatively narrow stride (i.e. feet set rather closely in front of the other) was congruent to that of the suspect 
the determination. In cases where the position of the head, and the specific moment in a gait cycle may be seen, for example, a stance phase, these error rates may be given more exactly (and with a smaller range) [11].
Attempting to show error rates for gait analyses and comparisons are also important. We recently compared gait angles for the hip, knee and ankle joints between 16 test subjects, and were able to determine the different phases of

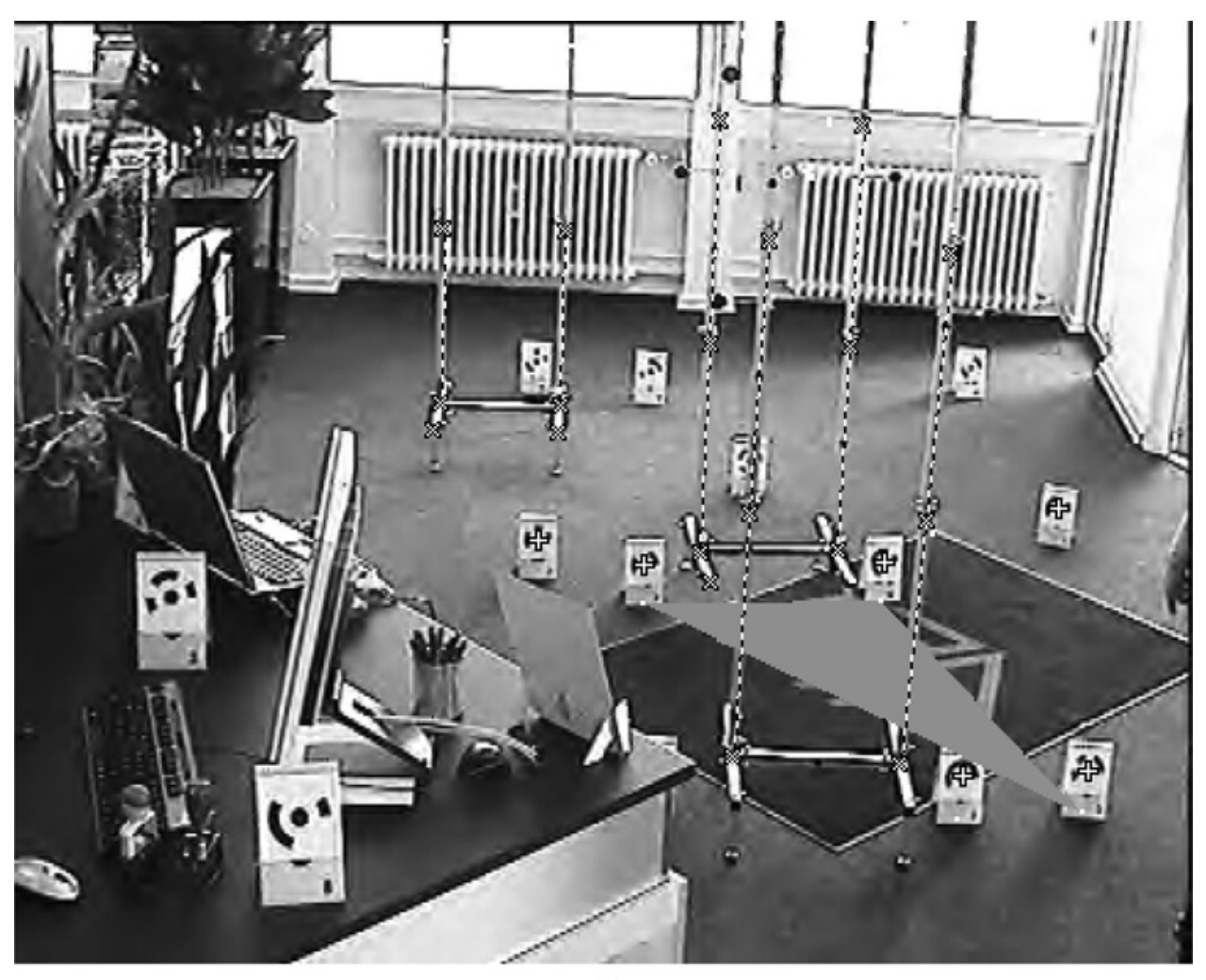

a

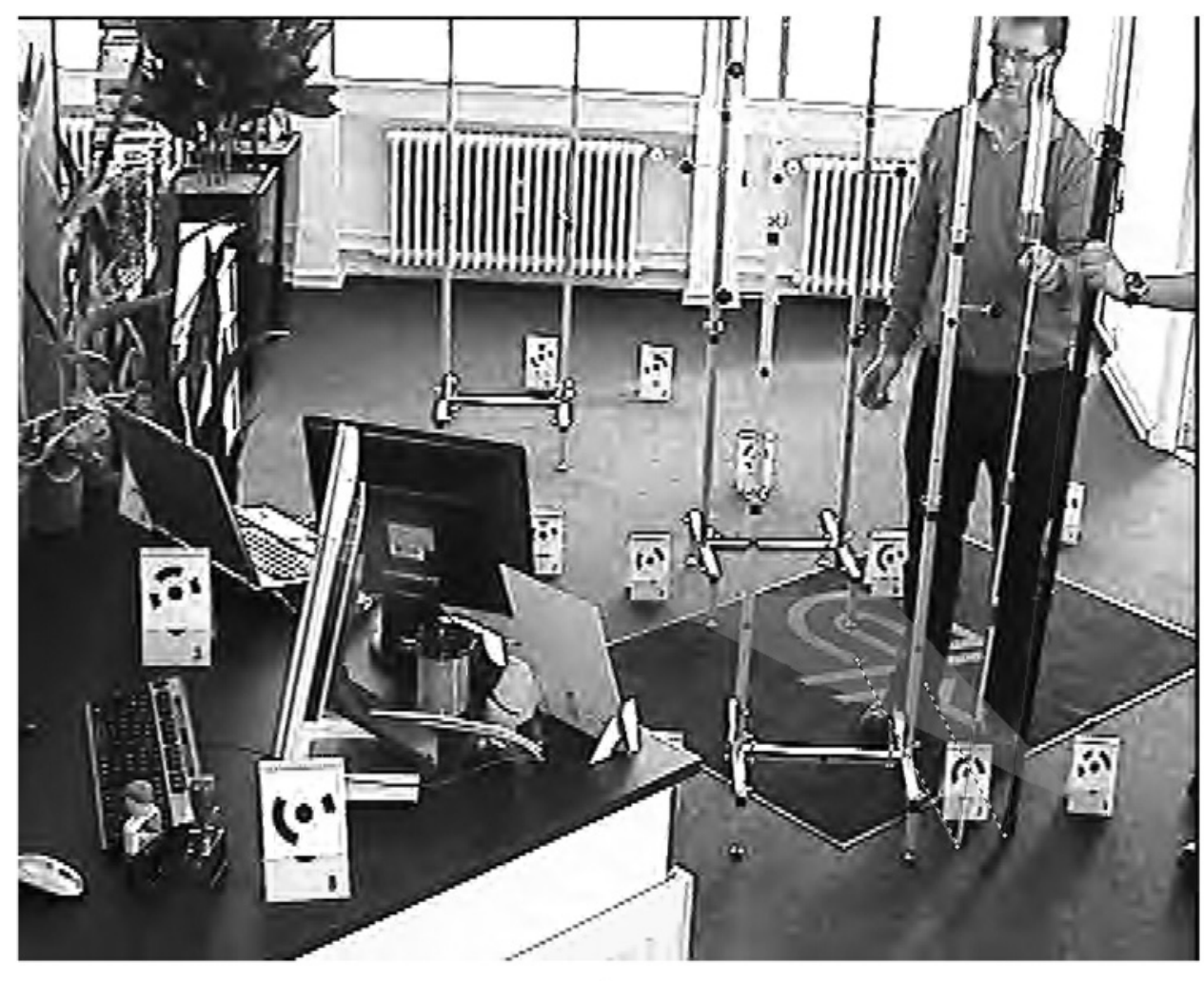

b

Fig. 4 Image incorporated in our reports in order to explain in a step-by-step fashion how photogrammetry is performed $a$ How measurement frames for camera and lens calibration are set up at the crime scene

$b$ How physical control of the measurement is done (cf. Fig. 2), by one of the authors holding a yardstick 
a gait cycle, where joint angles are very alike between the test subjects, and conversely those phases in a gait cycle, where the joint angles seem quite individual. In a real setting, where there is an image of a perpetrator and where the angles in the hip, knee or ankle joint can be assessed sagittally, one may then set these measurements against the phase of gait (by deducing from the image whether the perpetrator's leg is in the stance or swing phase), and thus give an opinion as to whether the angle measures are specific (and thus useful for comparison with images of a suspect), or very general, and thus not as useful for comparison [12]. We hope soon to implement the results of these tests in our case reports.

\section{Presenting evidence}

Gait and photogrammetric analyses are most often used to assess whether there may be a match between a perpetrator and one or more suspects. Ideally, such an analysis might conclude that there is a match, or identity has been proved, or no match, or identity may be disproved. However, in our opinion, such succinct conclusions cannot be given in these analyses. In our statements, we never use the words match or identification. We always write about 'congruence of identity', in order to underline the non-direct nature of the evidence. Even in the best of technical circumstances there may be a twin - a person with the same bodily features and measures, and a gait with many non-specific features.

Quite apart from the above-mentioned sources of error, even if the video footage was crystal clear, and even if a very conspicuous gait was recorded, it is still always data derived from a source. Unlike other forensic evidence, for example, alcohol blood testing performed on blood drawn from a specific individual, or DNA samples taken from a suspect, video and photographic evidence is not derived directly from the individual. Furthermore, and perhaps more importantly, in other forensic technical evidence, a database may be made in order to provide a statistical assessment of the specificity of a given match. This is, for example, the case in DNA profiling, where the match between the DNA pattern of a trace and the pattern from a suspect can be assessed in terms of how many other individuals could possess the same pattern, which in most cases can be calculated to be rarer than 1:1000000 [13]. There are no such databases at our disposition, perhaps excluding stature. By photogrammetrically calculating the stature of a suspect and comparing this to the stature of a suspect, both of which, for example, fall within $180-185 \mathrm{~cm}$, one can say, by referencing to national health databases, or databases compiled of students or conscripts, how many else in the population could have the same height. However, this is virtually impossible in terms of gait and bodily and facial features. How to tabulate the frequency of a pointed nose? The degree of bowleggedness? We find that this precludes offering precise statistical assessments, for example, in terms of a probability of a match, although this may change in the future [14].

When we analyse our cases, we always seek to state which features in our opinion may speak for a match, and which features may speak against a match. In some cases there may well be both. If no features speak either for or against, we write this in our statement. When we list certain features, we also give an assessment of whether the feature is a very general feature, or a more individualising feature. We have no solid statistical basis for this, but rely on our extensive experience in judging gait and from our scientific gait studies. For example, a perpetrator may have some degree of outward-pointing feet in the stance phase. While we also note this feature for the suspect, we state that outward-pointing feet is a general feature. On the other hand, a pronounced degree of hyperextension, or limping, may be said to be a more specific feature.

A case with two perpetrators robbing a bank may serve as an example of our case work approach. A suspect was subsequently apprehended. Based on the full video imagery from the bank CCTV system and from police surveillance recordings of the suspects (not shown), we compare the gait of one of the perpetrators with that of one of the suspects (we performed the full analyses so that each perpetrator was compared with each suspect). In this case, in one of the pairwise comparisons, we stated the following in our case report:

'The following general features of the gait were indicative of congruent identity:

- A gait rhythm characterised by 'heavy' strides.

- Degree and form of the lower leg forward extension before heel-strike (Fig. 3a).

- Degree of outward rotation of the foot in the stance phase, as seen from behind (Fig. $3 b$ ).

- A relatively narrow stride (i.e. feet set rather closely in front of the other) (Fig. 3c).

- A tendency for the upper body to be slightly backwardly flexed.

No features of the gait were contraindicative of congruent identity'.

The gait analyses thus comprises more general, combined, features of the gait, for example, 'heavy' and narrow strides, but also more specific features such as the foot rotation. Also, our gait analyses are always done by two observers, who have to agree on the features listed (as either indicative or contraindicative of congruence).

Conjunct to the above statements, we present the video materials where we could deduce these features, typically in built-in video files in a Powerpoint ${ }^{\circledR}$ stand-alone presentation package. The presentation may thus be seen directly from an accompanying $\mathrm{CD}$ and shown in court.

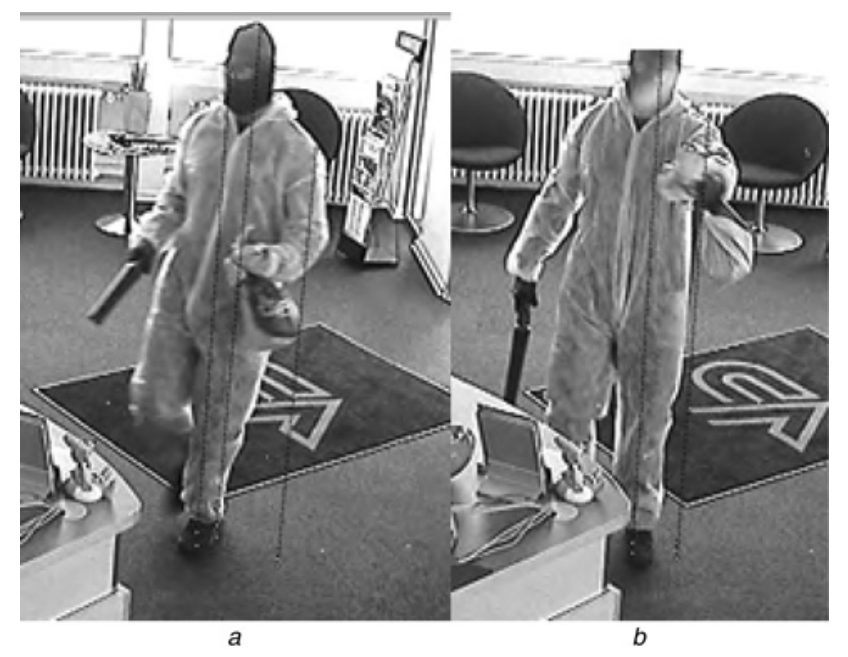

Fig. 5 After calibration, measurements may be done directly on the CCTV images

$a$ and $b$ The images show two situations (lines denote measurements) 
Table 1 Presentation of measurements of a perpetrator as given in our report (cf. Figs. $5 a$ and $b$ )

\begin{tabular}{lll}
\hline Measurement & Situation 1 & Situation 2 \\
\hline To top of black balaclava & $180-181 \mathrm{~cm}$ & $184-185 \mathrm{~cm}$ \\
To nose & $165-166 \mathrm{~cm}$ & \\
To shoulder & $146-147 \mathrm{~cm}$ & $155-156 \mathrm{~cm}$ \\
\hline
\end{tabular}

In the same case we also performed photogrammetric analyses of the height. In our case report, we included images of how the technique is used (Figs. $4 a$ and $b$ ), and how we arrived at these measurements (Figs. $5 a$ and $b$ ). In a table (Table 1), we presented the actual measurements, and to further illustrate error margins and to facilitate direct comparison with like measurements of one or more suspects, we included a graph (Fig. 6).

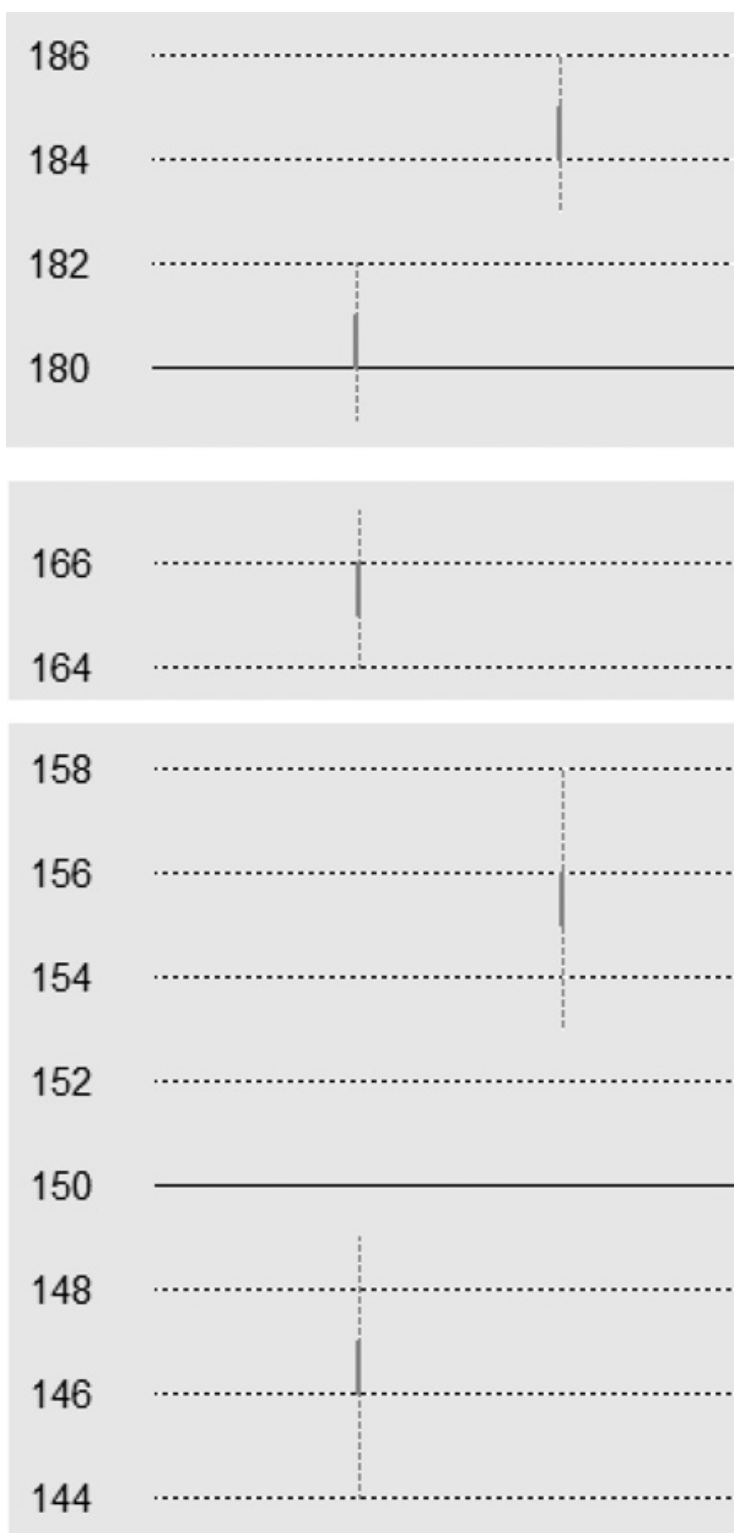

Fig. 6 Part of a graph included in our case report

The graph shows the measures in Table 1, with added bands for error margins. The righthand part of the graph has the same measurements for the suspect (not shown). From top to bottom the bands indicate the range of the measuremens in two situations: height to top of balaclava; nose hieght and shoulder height. Numbers denote $\mathrm{cm}$. It is seen that there is some difference in the measurements between the two situations
Table 2 Levels of evidentiary weight

A. Identification - When it is certain that the suspect has the same identity as the perpetrator

B. Strongly indicated - When several traits are found to point towards suspect and perpetrator having congruent identity C. Indicated - When few traits are found to point towards suspect and perpetrator having congruent identity

D. Cannot be excluded - When it is not possible to perform a comparison between suspect and perpetrator because of the (low) quality of the surveillance recordings

$E$. Very little speaks in favour of - When there is very little reason to believe that suspect and perpetrator have congruent identity F. Elimination - When it is certain that perpetrator and suspect cannot have congruent identity

Re-worked by the authors and based on the conclusion scale from ENFSI Expert Working Group Marks [15].

As a service for the police and the court, we further evaluate our evidence according to the guidelines set by the European Working Group Technical Evidence [15]. Basically, this working group has formulated a scale with five levels, covering the degree of match between a tool and tool marks (Table 2). For each case, we weigh the traits speaking for and against 'congruence of identity', and then chose the appropriate level, usually B or C. Following the above discussion, we will probably never be able to use levels $\mathrm{A}$ and $\mathrm{E}$. The level assignment is meant to align our evidence with other police technical evidence.

\section{Conclusion}

Photogrammetry and gait analyses may be used in forensics so that identification can be made when the more traditional identification approaches cannot be deployed. This requires analysis of the nature of evidence, the technical issues in the collection of that evidence and considerations about how to present the evidence and the conclusions in court. As for all forensic evidence, one should attempt to follow the guidelines established by the Daubert rules: in other words, being able to prove that the method is indeed scientific (i.e. has been subject to peer-review, published in relevant scientific journals), and that error rates can be assessed. Even though gait is probably highly individual, there are problems in being able to discern and measure the gait with a high enough resolution to capture this individuality. The state of the art, as presented here, does show that exact photogrammetric measurements may be made from CCTV material of perpetrators and at the scene of the crime, but that care must be taken to ensure that error ranges, especially connected to measuring the human (clothed and masked) body in motion, are critically estimated and evaluated. The future will probably bring automated systems with the ability to use markerless capture with a high degree of discrimination, even allowing for clothed subjects [16].

\section{References}

1 Thompson, T., Black, S. (Eds.): 'Forensic human identification: an introduction' (CRC Press, New York, USA, 2006) p. 544

2 Bouchrika, I., Goffredo, M., Carter, J., Nixon, M.: 'On using gait in forensic biometrics', J. Forensic Sci., 2011, 56, (4), pp. 882-889

3 Larsen, P.K., Simonsen, E.B., Lynnerup, N.: 'Gait analysis in forensic medicine', J. Forensic Sci., 2008, 53, (5), pp. 1149-1153

4 Sackett, D.L., Rosenberg, W.M., Gray, J.A., Haynes, R.B., Richardson, W.S.: 'Evidence based medicine: what it is and what it isn't', $B M J$, 1996, 312, pp. 71-72 


\section{www.ietdl.org}

5 Lynnerup, N., Vedel, J.: 'Person identification by gait analysis and photogrammetry', J. Forensic Sci., 2005, 50, (1), pp. 112-118

6 Larsen, P.K., Lynnerup, N., Henriksen, M., Alkjær, T., Simonsen, E.B.: 'Gait recognition using joint moments, joint angles, and segment angles', J. Forensic Biomech., 2010, 1, p. 7 (doi: 10.4303/jfb/F10032)

7 http://www.bailii.org/ew/cases/EWCA/Crim/2011/1296.html, accessed 8 January 2014

8 James, S.H., Nordby, J.J. (Eds.): 'Forensic science' (CRC Press, New York, USA, 2003)

9 http://www.bailii.org/ew/cases/EWCA/Crim/2012/1605.html, accessed 8 January 2014

10 Larsen, P.K., Hansen, L., Simonsen, E.B., Lynnerup, N.: 'Variability of bodily measures of normally dressed people using photo modeler ${ }^{\circledR}$ Pro 5', J. Forensic Sci., 2008, 53, (6), pp. 1393-9

11 Yang, S.X.M., Larsen, P.K., Alkjær, T., Juul-Kristensen, B., Simonsen, E.B., Lynnerup, N.: 'Height estimations based on eye measurements throughout a gait cycle', Forensic Sci. Int., 2014, 236, pp. 170-4
12 Yang, S.X.M., Larsen, P.K., Alkjær, T., Simonsen, E.B., Lynnerup, N.: 'Variability and similarity of gait as evaluated by joint angles: implications for forensic gait analysis', J. Forensic Sci., 2014, 59, (2), pp. 494-504

13 Aitken, C.G.G., Taroni, F.: 'Statistics and the evaluation of evidence for forensic scientists (Statistics in Practice)', (Wiley, 2004)

14 Reid, D., Nixon, M.S., Stevenage, S.V.: 'Soft biometrics; human identification using comparative descriptions', IEEE Trans. Pattern Anal. Mach. Intell., 2013, pp. 1-14, DOI: 10.1109/TPAMI.2013.219

$15 \mathrm{http}: / /$ www.poliisi.fi/intermin/hankkeet/wgm/home.nsf/files/Harmonized Conclusion_Scale_of_EWGM/\$file/Harmonized_Conclusion_Scale_of_ EWGM.pdf, accessed April 2013

16 Yang, S.X.M., Christiansen, M.S., Larsen, P.K., Alkjær, T., Moeslund, T.B., Simonsen, E.B., Lynnerup, N.: 'Markerless motion capture systems for tracking of persons in forensic biomechanics: an overview', Comput. Methods Biomech. Biomed. Eng., Imaging Vis., 2013. Online Available at: http://dx.doi.org/10.1080/21681163.2013.834800 